International Tinnitus Journal. 2018;22(2):93-96.

\title{
Translation of the Tinnitus Handicap Inventory (THI) into the Telugu language and Standardization
}

Rajendra Kumar Porika Balakrishnan Doraisami

\begin{abstract}
Tinnitus is estimated to be present in 10\%-15\% of the general population. The severity of tinnitus is judged based on the handicap, caused by it. Several tools have been developed for assessing the handicap level. One such tool is the Tinnitus Handicap Inventory (THI). This self-reporting questionnaire has 25 questions, in the English language. The questions describe common situations, covering three domains viz. functional, emotional and catastrophic. The patient has to answer 'Yes, No or Sometimes'. Globally, THI is accepted to have great utility, both in establishing the baseline and monitoring the efficacy of treatment. THI has been translated into several other languages. This article describes our endeavor to translate the same, into the Telugu language. We obtained due permission from the original author. In translation, a standard methodology was employed. The THI English version was translated into Telugu by two linguistic experts. The translated version was verified by experienced audiologists, for faithful reproduction to the original and for lucidity. Wherever required, the translation was suitably amended. All the professionals, above mentioned were native Telugu speakers, who were also proficient in English. Each question was checked with nontechnical lay persons, for easy comprehension and lucidity. Then, all the questions were back-translated into English, and checked for faithfulness. This Telugu version was christened as THI-T. This THI-T version was field tested and validated in 60 subjects with tinnitus, and in another 60 subjects, without tinnitus. Both the groups were provided with the Telugu version questionnaire and were asked to answer the questions. Statistical analysis were done using the Cronbach's Alpha test for internal consistency and test retest reliability was confirmed using the Pearson's 2 tailed correlation to determine statistical significance respectively. Results showed significant efficacy and reliability, of the translated THI-T tool.
\end{abstract}

Keywords: tinnitus handicap inventory, tinnitus, handicap, quality of life, questionnaire.

Department of Audiology, Ali Yavar Jung National Institute of Speech and Hearing Disabilities (Divyangjan) (AYJNISHD), Secunderabad, India Send correspondence to:

Rajendra Kumar Porika

Department of Audiology, Ali Yavar Jung National Institute of Speech and Hearing Disabilities (Divyangjan) (AYJNISHD), Secunderabad, India. E-mail: rajindrakumarp@ gmail.com

Paper submitted to the ITJ-EM (Editorial Manager System) on July 04, 2018; and accepted on July 24, 2018. 


\section{INTRODUCTION}

Tinnitus is the perception of sound within the human ear in the absence of corresponding external sound (Valente) caused due to abnormalities in the central nervous system with no associated mechanical, vibratory activity of the ear and other causes. Tinnitus has been poorly understood disorder, difficult to successfully diagnose and treat. Tinnitus Handicap inventory (THI) is a universally accepted tool which is used by many clinicians in their routine clinical practice to evaluate tinnitus severity. It provides valuable insight regarding difficulties faced by individuals suffering with tinnitus. It has been proven to be robust, psychometrically potent measure of evaluating tinnitus in everyday life situations and also evaluating outcome of many therapeutic used in everyday life. The present study designed to translate THI in Telugu language; therefore making easier to evaluate tinnitus in individuals those who speak Telugu.

\section{Assessment of the tinnitus}

Assessing the precise ways in which tinnitus affects you as an individual is an important first step in implementing a treatment regimen. The Good practice guide, published by the UK Department of Health in 2009, recommends that assessment of tinnitus should be done using a combination of structured interviews, audiological investigations and self- report questionnaires.

\section{Self-reporting questionnaires for assessment}

Any attempt to make an evidence based comparison of the efficacy of various regimens, is constrained by the heterogeneity of patients with tinnitus ${ }^{1}$. Each patient may exhibit a different combination of features. In this situation, Self- reporting questionnaires are very useful. Standardized and universally accepted grading tools are absolutely essential, in this context. A number of self-assessment tools have been designed by various authorities. A few commonly employed tools are mentioned here.

Tinnitus Reaction Questionnaire (TRQ) and Tinnitus Cognitions Questionnaire (TCQ): Wilson et al. designed a TRQ and a TCQ, based on the cognition theory. Both questionnaires are self-reporting, and have 26 questions, each. Each of the questions asks about the responses of the individual patient, to tinnitus, in various real life situations ${ }^{2}$. The TRQ items cover the (i) emotional reactions, (ii) interference with work and sleep, and (iii) the feelings that are evoked due to tinnitus. The TCQ is based on the Cognition Theory. Out of the total of 26 questions, one half pertains to positive thoughts, which arise in your mind, in response to tinnitus. An equal number of questions pertain to negative thoughts.

TFI (Tinnitus Functional Index): Miekle et al. published this scale, under funding from the Tinnitus Research Consortium of the United States. This was new outcome measure was found to be (i) highly sensitive to treatment effects i.e. 'responsiveness' (ii) addressing all major dimensions of tinnitus impact and (iii) validated for scaling the negative impact of tinnitus. Overall, the TFI performed well in the RCT with Cohen's $d$ being 1.23. Comparison of TFI performance was made with the Tinnitus Handicap Inventory ${ }^{3}$. All of the results confirmed sensitivity of the TFI along with its subscales. Since then the TFI has received increasing international use and is being translated into several languages, across the globe.

Tinnitus Handicap Inventory (THI) is another tool developed in 1966, by Newman et al. initially as an alpha version with a 45 item scale. After some initial trials, a beta version was refined by the same team, with a reduced number of 25 questions. The 25 questions cover three domains viz. functional, emotional and catastrophic ${ }^{4}$. The subject has to answer each question with any one of three options-Yes, sometimes or No. These options are assigned scores of 4,2 , and 0 points, respectively. An aggregate maximum score of 100 is possible. An aggregate score of ' $O$ ' indicates no perceived handicap and ' 100 ' would indicate maximal handicap. This THI beta is a self-report measure that can be used in a busy clinical practice to quantify the impact of tinnitus on daily living. This tool has found global acceptance and is a very popular one.

\section{Translations of Tinnitus Handicap index (THI), in different languages}

THI was first developed in English. Over the years, it has been translated into several languages including German, Dutch, Spanish, Portuguese, Turkish, Danish, Chinese - Cantonese, Thai, and Korean ${ }^{5-10}$. Within India, $\mathrm{THI}$ has been translated into Kannada, Malayalam and Tamil languages. The Kannada version was developed by Zacharia et al. and field tested in 140 subjects $^{11}$. The Malayalam language version was developed by Aithal et al. and field tested with 50 tinnitus patients and 40 controls ${ }^{12}$. The Tamil translation was done by Ramkumar and Swaminathan in 2015, and validated with 100 subjects $^{13}$.

\section{Objective}

Our objective is to translate this globally acclaimed tool into the Telugu language. This will enable its usage, across the native Telugu population. We christened the Telugu language version the Tinnitus Handicap Inventory as THIT

\section{METHODOLOGY, FOLLOWED IN DEVELOPING TINNITUS HANDICAP INVENTORY IN TELUGU (THIT)}

The THI has passed the tests of time and several studies. Hence, we decided to use the entire set of 25 questions and, thus preserve the construction. In our endeavor, we followed the standard World Health Organization's (WHO) (2018) guidelines on the Process of translation and adaptation of instruments.

As the first step, two teams were formed: one 
translation team and one bilingual team. The Translation team consisted of two linguistic experts, who were proficient in Telugu and in English. The Bilingual team consisted of four members, who were native Telugu speakers and proficient in English also. Three of the four, were practicing audiologists, who were well aware of THI in its English version. The fourth person was a nontechnical lay person. He was bilingual ${ }^{14,15}$.

\section{Initial translation}

The translation team translated the THI English version into the commonly spoken Telugu language. Telugu is a well-developed language with great literature and music. The translators took special care to avoid the refined language of the hoary literature.

\section{Verification of the translation}

The Bilingual verified the initial translation to ensure that the translated version was faithful to the original version and that there were no ambiguities or alterations in the meaning of questions and accompanying explanations. Wherever needed suitable changes were made in consultation with the translation team.

\section{Back translation}

After the above, one independent Telugu professor, who was proficient in both Telugu and English did a back translation. He was not a member of the initial translation team, and was blind to them. This back translation was read by the bilingual team. They made suitable refinements to the translated version.

\section{Testing for comprehension by native Telugu speakers}

The Telugu version, obtained as above, was given to ten native Telugu lay persons, who were monolingual and did not know English. Out of the ten, five subjects had tinnitus and five did not have any tinnitus. This session was carried out in the presence of at least one translator and at least one bilingual member. Wherever the monolingual subjects did not comprehend or expressed doubts, the version was appropriately changed. This ensured that the Telugu version had fidelity to the original and additionally, it was appropriately understandable to the lay Telugu speakers.

\section{Field testing pilot study}

This above refined questionnaire was administered to sixty individuals with Tinnitus (case group) and another sixty individuals without tinnitus (control group). Their ages ranged from 16 years to 75 years.

In the control group of normal persons, we made sure that there were no hearing problems, or tinnitus, by a detailed history. This absence of hearing related issues was confirmed by doing a basic test battery of pure tone audiometry, impedance test and oto acoustic emission test. The case group of tinnitus patients, in addition to the above basic test battery, had specific tests for tinnitus viz. pitch matching test and loudness matching test.

After this, both the groups were provided with the Telugu questionnaire and were asked to answer the questions. Based on their scores, the severity of their handicap was graded, by the classification given in Table 1.

Statistical analysis included the use of the following tests viz. the Cronbach's Alpha test for internal consistency. Also, test retest reliability was confirmed using the Pearson's 2 tailed correlation to determine statistical significance respectively.

\section{RESULTS}

\section{Sample characteristics}

The THIT was administered to a total of 120 subjects (sixty persons with tinnitus and sixty persons without tinnitus). Their age and gender profile is given in Table 2 and the overall THIT severity of the tinnitus group levels have been shown in Table 3.

\section{Item analysis}

The Item analysis was carried out for THIT tool by computing item-total correlations (Table 4) for tinnitus group $(\mathrm{N}=60)$. The correlation between each item with total score of the scale have shown a very high significant correlation $(p<0.01)$; indicating the validity of the each item included in the tool.

The internal consistency of the tool was analyzed by Cronbach's Alpha (Table 5). A very high internal consistency was established $(\alpha=0.981)$ indicating the overall efficacy of the inventory when administered amongst the 120 individuals. Also test-retest Pearson's two tailed correlation also showed a high reliability 0.99

Table 1. Rating scale used in classification of the individuals, by their THI scores.

\begin{tabular}{lc}
\hline THI score & Category \\
\hline $0-16$ & No handicap \\
$18-36$ & Mild handicap \\
$38-56$ & Moderate handicap \\
$58-100$ & Severe handicap \\
\hline
\end{tabular}

Table 2. Age and gender of the sample.

\begin{tabular}{lccccc}
\hline \multirow{2}{*}{ Group } & \multicolumn{3}{c}{ Age (years) } & \multicolumn{2}{c}{ Gender } \\
\cline { 2 - 6 } & N & Mean Age & SD & Male & Female \\
\hline With Tinnitus & 60 & 47.5 & 15.8 & $36(60 \%)$ & $24(40 \%)$ \\
Without tinnitus & 60 & 23.1 & 6.59 & $31(51.6 \%)$ & $29(48.3 \%)$ \\
\hline
\end{tabular}

Table 3. THIT severity of the tinnitus group.

\begin{tabular}{lcc}
\hline Severity & Frequency & Percentage \\
\hline No handicap & 15 & 25 \\
Mild handicap & 6 & 10 \\
Moderate handicap & 21 & 35 \\
Severe handicap & 18 & 30 \\
Total & 60 & 100 \\
\hline
\end{tabular}


Table 4. Item-total correlations THIT tool in tinnitus group.

\begin{tabular}{|c|c|}
\hline Items & Pearson -'r' \\
\hline Q1 & $0.720^{* *}$ \\
\hline Q2 & $0.664^{* *}$ \\
\hline Q3 & $0.770 * *$ \\
\hline Q4 & $0.797^{\star *}$ \\
\hline Q5 & $0.746^{\star *}$ \\
\hline Q6 & $0.787^{\star *}$ \\
\hline Q7 & $0.730^{* *}$ \\
\hline Q8 & $0.771^{* *}$ \\
\hline Q9 & $0.771^{\star *}$ \\
\hline Q10 & $0.687^{\star *}$ \\
\hline Q11 & $0.739 * *$ \\
\hline Q12 & $0.769 * *$ \\
\hline Q13 & $0.707^{\star \star}$ \\
\hline Q14 & $0.678^{* *}$ \\
\hline Q15 & $0.542^{\star \star}$ \\
\hline Q16 & 0.721 ** \\
\hline Q17 & $0.668^{\star *}$ \\
\hline Q18 & $0.570^{* *}$ \\
\hline Q19 & $0.672^{\star \star}$ \\
\hline Q20 & $0.729 * *$ \\
\hline Q21 & $0.737^{\star \star}$ \\
\hline Q22 & $0.800^{\star *}$ \\
\hline Q23 & 0.760 ** \\
\hline Q24 & $0.675^{\star \star}$ \\
\hline Q25 & $0.747^{\star \star}$ \\
\hline$\star *-p<0.01$. & \\
\hline
\end{tabular}

Table 5. Reliability analysis-Cronbach's $\alpha$.

\begin{tabular}{lcc}
\hline Group & N & Alpha (a) \\
\hline Tinnitus & 60 & 0.961 \\
Without Tinnitus & 60 & 1 \\
Overall & 120 & 0.981 \\
\hline
\end{tabular}

Table 6. Test-retest reliability-Pearson-' $r$ '.

\begin{tabular}{lcc}
\hline Group & N & Pearson-r \\
\hline Tinnitus & 60 & 0.981 \\
Without Tinnitus & 60 & 1 \\
Overall & 120 & 0.999 \\
\hline
\end{tabular}

and the said inclusion of both the groups if we look into the Table 6. A significance of 0.01 was established showing good reliability of the THI.

\section{DISCUSSION}

The Tinnitus Handicap Inventory is a universally accepted tool, to assess the degree of handicap in an individual's personal lifestyle. The THI was translated by us, by following standard procedures viz. translation, back translation, team approach, consideration of the special nuances of the native language and a pilot field study in clinical settings. The fidelity of the translated version was ensured by this standard procedure. A high significant Cronbach's alpha $(\alpha=0.98)$ was established for internal consistency of this tool developed for Telugu language.

\section{CONCLUSION}

The present study aimed to develop and standardize an adaptation of THI in Telugu language, making it easier to administer the same to the Telugu speaking individuals. This Telugu version has passed the statistical tests. The pilot study has also established the robustness of THIT viz. the Telugu version of the Tinnitus Handicap Inventory.

\section{ACKNOWLEDGEMENT}

Tinnitus Handicap Inventory is an universally acclaimed tool, utilized globally, in English and in various other languages. The original English language version had been developed by Newman Craig W, Jacobson GP and Spitzer JB in the year 1996. The authors gratefully acknowledge the readiness, with which he gave us permission to translate the same to English. This, our, work had been made possible, only by his encouragement.

\section{REFERENCES}

1. Baguley D, McFerran D, Hall D. Tinnitus. Lancet. 2013;382:16001607.

2. Wilson PH, Henry J, Bowen M, Haralambous G. Tinnitus reaction questionnaire: psychometric properties of a measure of distress associated with tinnitus. J Speech Hear Res. 1991(1):197-201.

3. Meikle MB, Henry JA, Griest SE, Stewart BJ, Abrams HB, McArdle $R$, et al. The tinnitus functional index: development of a new clinical measure for chronic, intrusive tinnitus. Ear Hear. 2012;33(2):153-76.

4. Newman CW, Jacobson GP, Spitzer JB. Development of the tinnitus handicap inventory. Arch Otolaryngology Head Neck Surg. 1996;122(2):143-8.

5. Shaurya C, Ravichandran A, Kumar S. Transadaptation and standardization of tinnitus primary function questionnaire in Hindi. Int Tinnitus J. 2018;22(1):23-29.

6. Schmulian D. The Clinician Managing Tinnitus Distress: A preliminary study in clinical engagement. Int Tinnitus J. 2018;22(1):46-53.

7. Adegbiji WA, Olajide GT, Olubi O, Aluko AAA, Olajuyin AO, Eletta $\mathrm{PA}$, et al. Characteristics and management of childhood tinnitus in a developing country. Int Tinnitus J. 2018;22(1):66-71

8. Pinkl J, Wilson MJ, Munguia-Vazquez R, Billingsly D. Detailed analysis of high frequency auditory brainstem response in patients with tinnitus: A preliminary study. Int Tinnitus J. 2017;21(1):35-43

9. Frosch FG. Possible joint involvement of the cochlea and semicircular canals in the perception of low-frequency tinnitus, also called "the hum" or "taos hum". Int Tinnitus J. 2017;21(1): 63-67

10.Rikitake M, Sampei S, Komori M, Sakura Y, Sakura H. Bilateral deafness as a complication of the vaccination-A case report. Int Tinnitus J. 2018;22(1):19-22

11.Zacharia T, Naik PV, Sada S, Kuniyil JG, Dwarakanath VM. Development and standardization of tinnitus handicap inventory in Kannada. Int Tinnitus J. 2012;17(2).

12. Aithal VU, Pillai VV, Zacharia T, Rajashekhar B. Development and standardization of tinnitus handicap inventory in Malayalam. Int Tinnitus J. 2013;18(2):129-133.

13. Ramkumar V, Swaminathan S. Validity and reliability of a tamil translation of the tinnitus handicap inventory. JISH. 2015;25(2):122127.

14. Henry JA, Griest S, Thielman E, McMillan G, Carlson KF. Tinnitus functional index: development, validation, outcomes research, and clinical application. Hear Res. 2016;334:58-64.

15.World Health Organisation (WHO). Guidelines on the process of translation and adaptation of instruments 2018. 